\title{
Vestigios poéticos y pervivencia del teatro litúrgico en el siglo XVI
}

\author{
Poetic Vestiges and Survival \\ of Liturgical Drama in the $16^{\text {th }}$ Century
}

\author{
Miguel Carabias Orgaz \\ IES El Greco (Toledo)
}

\section{RESUMEN}

Presentamos una serie de textos poéticos contenidos en un manuscrito de la segunda mitad del siglo XVI, los cuales se ponen en relación con ciertas manifestaciones dramáticas y procesionales que contribuyeron al desarrollo del teatro litúrgico castellano de los siglos XV y XVI. Este tipo de composiciones en lengua romance aparecieron con frecuencia interpoladas en el oficio, siguiendo cierto orden teatral, y pervivieron incluso después de que las disposiciones tridentinas impusieran la depuración de dichos materiales y la prohibición de aquel tipo de representaciones.

Palabras Clave: Teatro litúrgico, lírica tradicional, vueltas a lo divino, oficio, Ordo Prophetarum.

\begin{abstract}
We present a series of poetic texts included in a manuscript from the second half of the $16^{\text {th }}$ century, which are related to certain theatrical and processional manifestations that contributed to the development of the $15^{\text {th }}$ and $16^{\text {th }}$ century Castilian liturgical drama. Such compositions in the Romance language often appeared interjected in the offices, following a certain theatrical order, and persisted even after the Tridentine decisions imposed the purification of those lines and the prohibition of that sort of performances.
\end{abstract}

Key words: Liturgical drama, traditional poetry, contrafacta, offices, Ordo Prophetarum.

\section{LÍRICA ROMANCE Y TEATRO LITÚRGICO}

Al estudiar la literatura de la Edad Media, la crítica ha venido incidiendo durante los últimos años en el carácter impreciso de las fronteras que separan los diversos géneros y formas, especialmente en lo que se refiere al teatro medieval, donde el límite entre lo dramático y lo lírico, y aun entre la misma literatura y otras disciplinas artísticas como la música, se desdibuja en 
un amplio contexto cultural cuyo principal nexo de unión parece hallarse en la liturgia.

En este sentido, al analizar la relación entre el rito y el texto dramático, Turner (1986) ya señaló que lo ritual era concebido en la Edad Media como performance o representación, y no como un mero conjunto de reglas. El teatro, por su parte, sería el resultado de una «exageración de procesos jurídicos y rituales», desarrollándose en un contexto de espectáculo religioso, fundamentalmente litúrgico y musical, y en un espacio que cabría calificar de para-dramático.

Más recientemente, el profesor Pedro Cátedra (2005) se ha ocupado ampliamente de esta relación entre liturgia, poesía y teatro en la literatura medieval. Ha planteado que, sobre la práctica litúrgica, se fueron interpolando breves fragmentos poéticos romances, inicialmente por medio de traducciones directas de los textos litúrgicos, posteriormente a través de obras creadas ex novo para el mismo uso, todo ello como resultado de una «hipertrofia de la misma liturgia latina y gracias a una necesidad cultural y funcional».

De este modo, ciertas costumbres litúrgicas como las de Navidad o Semana Santa terminaron abriéndose a prácticas complementarias en las que se incorporaría la lengua vulgar, alternando elementos musicales y poéticos en lengua romance (Cátedra, 2000). Tal como apuntó ya Donovan (1958), la liturgia terminaría modificándose al servicio de interpolaciones dramáticas.

El resultado fue un conjunto de poemas y canciones dispuestos con algo de orden teatral, y cuya inserción en los oficios implicaría una cierta «representación». Por tanto, algunas piezas castellanas, como las de Gómez Manrique o Álvarez Gato, serían elementos destinados a insertarse en la práctica litúrgica, pues ante todo primaba la condición útil de aquélla (Surtz, 1983). En todos estos casos, las piezas tendrían un destino funcional, pedagógico.

Coincidiendo con el impulso de la poesía religiosa castellana del último cuarto del siglo XV y con el nutrido corpus musical de piezas navideñas, se produjo un importante desarrollo dramático del oficio, dando lugar a un teatro navideño en los siglos XV y XVI. Este desarrollo sería paralelo a la inserción de piezas en lengua romance, las cuales a su vez se irían distanciando paulatinamente del ámbito de la liturgia.

Por otro lado, el profesor Cátedra apunta que, aunque dicho teatro litúrgico tuvo su desarrollo dentro de una «tradición cerrada» de ámbito monástico o catedralicio y estrechamente vinculado a la liturgia, esto no impidió una cierta permeabilidad (Cátedra, 2005: 24). En efecto, las relaciones entre el dominio religioso y el laico serían habituales, utilizándose en la elaboración de estas piezas teatrales poemas y canciones de origen tradicional. En este sentido, cabe considerar la incidencia que hubieron de tener las denominadas «vueltas a lo divino».

A lo largo del siglo XVI, sin embargo, al amparo de la normalización tridentina, se irá produciendo un paulatino desplazamiento y depuración de los 
materiales romances. Hacia mediados del siglo se habían prohibido ya las representaciones en diversas catedrales españolas y, al mismo tiempo que el oficio perdía su carácter dramático, se avanzaba en la completa desaparición del teatro litúrgico, que sería sustituido, desde finales de siglo, por otros elementos como el canto de «villancicos» en las iglesias. Pero algunas de aquellas piezas dramáticas en lengua romance quedarían fosilizadas, y la poesía de Navidad, al cabo del tiempo, perduraría como testimonio. De ahí la necesidad de volver a plantearse el estudio de ciertos textos literarios, especialmente textos líricos, en tanto que puedan estar dotados de una determinada carga de teatralidad (Sirera, 1992).

En este punto cabe situar los textos que presento a continuación, dos poemas recogidos en la segunda mitad del siglo XVI, pero que sin duda responden a una tradición anterior, hundiendo sus raíces en aquellas piezas teatrales del XV, que tendrían sus derivaciones directas en la siguiente centuria. Mi intención no es otra que la de dar a conocer estos que considero vestigios de una práctica dramática y procesional por entonces casi extinguida. Ciertamente no son de una gran calidad literaria, pero en apenas cuarenta versos se puede intuir un corpus poético mucho mayor, seguramente ordenado de forma coherente en torno al oficio navideño.

\section{EL MANUSCRITO}

Los poemas que presento se hallan insertos en un protocolo notarial depositado en el Archivo Histórico Provincial de Salamanca bajo la signatura 4305. Se trata de un copioso volumen sin paginar que contiene documentación fechada entre los años 1576 y 1580, perteneciente a la escribanía de Esteban Carnicero, escribano público del número y concejo del lugar de Huerta, jurisdicción de la ciudad de Salamanca. Concretamente, forman parte de un cuadernillo compuesto por dieciocho folios que contienen la partija de los bienes de Marina Barbera, vecina de la aceña de San Bricio ${ }^{1}$, jurisdicción de la ciudad de Salamanca, y que lleva la fecha de 21 de abril de 1576. Los poemas quedaron anotados en uno de los folios sobrantes.

El texto, que transcribo más abajo, fue copiado por la misma mano que el resto de la documentación notarial del protocolo, en letra procesal del siglo XVI, presentando abundantes enlaces y una gran irregularidad en cuanto

${ }^{1}$ La aceña de San Bricio, hoy desaparecida, se ubicaba al norte del actual término municipal de Huerta (Salamanca). La documentación del siglo XVI citaba, además, una ermita de San Bricio, que hubo de situarse muy cerca de allí, y que nos trae al recuerdo a aquella ermitaña celestinesca de la égloga de Lucas Fernández, una obra en la que son frecuentes los topónimos salmantinos. Puede consultarse al respecto la documentación del AHPS, Protocolos, 4305 y 4306. 
a la separación de palabras. No obstante, el escribano Esteban Carnicero no parece haber sido su autor, sino un mero copista. Entre otras cosas, hay ciertos errores de copia que lo delatan: el más significativo es que confunda el nombre de Jesé, tal vez extraño para él, con el de José, personaje bíblico mucho más conocido. También puede observarse que Carnicero había copiado algunos versos de una estrofa anterior, pero los tachó luego y añadió los versos correctos por encima.

Aunque el estado de conservación del manuscrito es bueno, las diversas correcciones y tachaduras, así como la precipitación con que fueron copiados los poemas, dificultan su lectura. El copista deja en ocasiones incompletos los versos que se repiten en el estribillo. Da la impresión de que nos hallamos ante un documento escrito, no con la finalidad de ser conservado, sino más bien con la intención de ser «utilizado» de manera puntual e inmediata.

\section{OBSERVACIONES SOBRE LA LENGUA. CRITERIOS DE TRANSCRIPCIÓN}

En primer lugar, cabe destacar la presencia de algunos arcaísmos. Éstos podrían ponerse en relación con textos preexistentes o con la fusión de elementos textuales diversos. Llama la atención la $-e$ paragógica de la palabra rayze, que cabría explicarse, tal como sugiere Lapesa (1981: 122), como un rasgo arcaizante presente en la lírica castellana de los siglos XV y XVI, documentándose testimonios análogos como voze, luze, virtude, etc. En todo caso, como veremos, la métrica del poema sugiere que no tendría valor fonológico. También podría considerarse como arcaizante la forma enclinando, que alternó en el castellano medieval con la más culta inclinando (ĬNCLĪNARE > inclinar), y que desde el siglo XVI fue desplazada en la lengua literaria por la forma culta (Corominas, 1980: III, 445).

Por otro lado, la presencia de cultismos, si no importante en cuanto a su número, resulta empero significativa si la ponemos en relación con el contexto literario al que ya hemos hecho referencia. Es paradigmático el uso de alguna forma léxica del ámbito eclesiástico: el adjetivo vera, que fue sustituido desde el siglo XIV por verdadera, tal y como señala Corominas (1980: V, 789), sería de nuevo frecuente como cultismo en los siglos XV y XVI en «el habla medio latina de los clérigos».

Cabe, finalmente, señalar la presencia de ciertos rasgos dialectales que pueden ayudarnos a ubicar geográficamente el texto. Encontramos un ejemplo de metátesis en perguntan, probablemente por influjo del portugués, y que puede encontrarse también en las obras de Gil Vicente. Se utiliza en el texto la forma nun para el indefinido masculino singular, que fue identificada por Gonzalo Correas (1967: 387) como propia de las hablas sayaguesas; e igualmente se ha relacionado con el habla sayaguesa la ausencia de $-d$ final en algunas palabras (voluntá), especialmente en el uso del imperativo, producién- 
dose aquí incluso cuando sigue un pronombre enclítico (aguardame). En este mismo sentido, cabe destacar el uso de la forma verbal ajuntan, que Corominas (1980: III, 539) señaló como «rara» en castellano, y que él mismo ponía en relación con las hablas salmantinas y asturianas. Todo ello parece apuntar, por tanto, a un contexto dialectal occidental, hecho que puede observarse también en buena parte de nuestros autores dramáticos de la primera mitad del siglo XVI, como Juan del Encina, Lucas Fernández o Gil Vicente (LópezMorales, 1967), y que no necesariamente ha de suponer la deliberada recreación de un estereotipo de lenguaje rústico.

He optado por una transcripción fiel del texto, respetando la alternancia gráfica de $i$ / $j$ / y en posición vocálica, así como la de $q$ / $c$ para representar la oclusiva velar sorda. No obstante, sigo los criterios modernos de acentuación y uso de mayúsculas, según la Real Academia Española. Separo las palabras según las normas actuales y resuelvo por medio de cursiva las abreviaturas, que son abundantes ( $q$, aql, qteys, porq, vra, lamentādo, etc.). Aquellas palabras o versos que el copista dejó incompletos los suplo entre corchetes, y reproduzco tal cual los versos tachados.

\section{TEXTO}

$\begin{array}{ll} & {[\mathrm{I}]} \\ \text { De la rayze de Jesé } & \\ \text { naze la virgen que fue } \\ \text { la más alta que yo sé, } \\ \text { que parió } \\ 5 \quad \text { al mysmo que la crió. } \\ \text { De la rayze naze planta, } \\ \text { de la planta se levanta } \\ \text { una symiente que planta, } \\ \text { que parió } \\ \text { al mysmo que la crió. } \\ \\ \\ \text { [II] } \\ \text { Los profetas me demandan: } \\ \text { - Señor, j quando vendréys? } \\ \text { - Aguardame, pecadores, } \\ \text { que muy p[r]esto me veréys. } \\ \text { El profeta Jeremýas } \\ \text { está contino llorando }\end{array}$

\footnotetext{
${ }^{2}$ Como ya he apuntado, en el manuscrito se lee José, lo cual es sin duda un error de copia, pues hace referencia al tronco o estirpe de Jesé, del cual desciende Cristo y, según cierta tradición, también la Virgen.
} 
y en nun monte lamentando

todas las noches y días,

y responden sus porfías:

10

-My Señor, pues que podéys.

-Aguardame, pecadores,

que muy $\mathrm{p}[\mathrm{r}]$ esto me veréys.

Aquel p[r]ofeta David

en un salmo está rogando

y a los cielos enclinando que tiene al mando de sí. my señor pues que podéys

-Vos mysmo, Señor, decí,

agtardame peadores

nuestra voluntá quitéys.

que ntmy $\mathrm{p}[\mathrm{r}] \mathrm{es}$

-Aguardame, pecadores, que muy [presto me veréys].

A mý sólo lo pergunta[n] porque vera yglesia soy, yo por respuesta les doy que los tiempos ya se ajuntan.

25 - Myra, Señor, que os barruntan

a lo que me respondéys.

- Aguardame pecadores que muy $\mathrm{p}[\mathrm{r}] \mathrm{es}[$ to me veréys].

\section{FORMAS, TEMAS, CONTEXTO LITERARIO}

\section{[I] De la rayze de Jesé}

El primero de los poemas, compuesto por dos estrofas, presenta una estructura de canción en trísticos monorrimos sin vuelta, a los que sigue un estribillo que se repite en forma de dístico también monorrimo, según la técnica de la represa. Se inserta, como artificio, el pie quebrado en el primer verso de dicho estribillo. No aparecen, sin embargo, los versos iniciales, lo cual nos hace pensar que quizá el copista decidió obviarlos por ser ya sobradamente conocidos. El esquema métrico sería el siguiente: $a^{8} a^{8} a^{8} b^{4} b^{8}$.

Destaca en el poema, ante todo, la regularidad métrica, alternando el verso octosílabo con el pie quebrado. Dicha regularidad no se ve afectada, en el primer verso, por la palabra rayze, cuya - e paragógica, como ya queda comentado, no tendría valor fonológico. No se hace uso de licencias métricas, ni siquiera de la sinalefa. Predominan los versos oxítonos y la rima aguda, y son pocos los versos y rimas paroxítonos. La rima es consonante perfecta, salvo en fue y parió, y resulta empobrecida al apoyarse en la homonimia de planta, en los versos sexto y octavo. 
El ritmo del poema viene marcado por fuertes paralelismos, no sólo métricos, sino también estilísticos: destaca el uso de la anáfora y la reiteración del pronombre relativo que, y hallamos un ejemplo de anadiplosis en los versos 6 y 7 .

Se incide, además, en el uso de la paradoja, tan habitual en la literatura religiosa. Concretamente, la idea aquí apuntada a modo de estribillo («que parió / al mysmo que la crió») será muy utilizada en la lírica religiosa desde finales del siglo XV. Fray Iñigo de Mendoza, por ejemplo, se valía de ella en sus Coplas de Vita Christi:

$$
\begin{aligned}
& \text { ¡O tan celestial muger } \\
& \text { que en el mundo meresçió } \\
& \text { sin dexar de virgen ser } \\
& \text { ver de sí mesma nasçer } \\
& \text { al mismo que la crió! }
\end{aligned}
$$

Mediado el siglo XVI, en el cancionero de Uppsala, podemos encontrar la siguiente composición, que contiene además un latinismo de clara resonancia eclesiástica:

$$
\begin{aligned}
& \text { Gózate, Virgen sagrada, } \\
& \text { pues tú sola merecistes } \\
& \text { ser madre del que paristes. } \\
& \text { Oh bendita sin medida, } \\
& \text { madre del que te crió, } \\
& \text { ante secula escogida } \\
& \text { de Dios, que de ti nasció, } \\
& \text { a madre jamás se dio } \\
& \text { la gracia que tú tuvistes; } \\
& \text { ser madre del que paristes. }
\end{aligned}
$$

Algunos años después, en las primeras décadas del siglo XVII, Baltasar Elisio de Medinilla recogía los siguientes versos para glosarlos en su villancico «A la Natividad de la Virgen Nuestra Señora», y que por tanto nos remiten a una composición anterior:

Hoy la tierra nos ha dado una flor, que desde el suelo a su olor traerá del cielo al mismo que la ha criado.

Por otro lado, la utilización que se hace en nuestro poema de la alegoría bíblica del árbol y la raíz de Jesé en relación con la Virgen no es en absoluto casual. Esta alegoría se pone en directa relación con el texto bíblico y con una tradición litúrgica concreta, lo cual apunta al contexto ritual o procesional en que seguramente se insertaban estos poemas. El texto latino de la Vulgata en el libro de Isaías (Egreditur virga de radice Iesse, et flos de radice eius as- 
cendet) presenta deliberadamente la forma virga, e inspira el texto litúrgico de diversas celebraciones marianas como el oficio del día de la Anunciación:

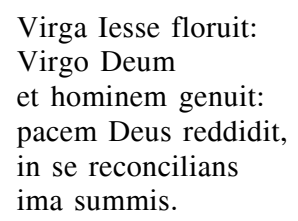

Estos versos, además, se ponen en directa relación con el primer teatro litúrgico europeo, concretamente con el Ordo Prophetarum, que en las versiones de San Marcial de Limoges, Laon y Rouen presenta la siguiente intervención del profeta Isaías:

\author{
Est necesse \\ Virgam Iesse \\ De radice provehi \\ Flos deinde \\ Surget inde \\ Qui est filiu Dei
}

En la poesía castellana, la referencia más antigua a la raíz de Jesé en relación con la genealogía mariana la encontramos en Loores de Nuestra Seño$r a$, de Gonzalo de Berceo, obra en la que, por cierto, se ha querido adivinar alguna reminiscencia del Ordo Prophetarum (Marchand, 1984):
En ti s'cumplió, Señora, el dicho d'Isaía: que de radiz de Yessé una verga saldría, e flor cual non fue vista Spíritu con siet'dones dend'se levantaría, en la flor posaría.

Son más claras las analogías que encontramos entre nuestro poema y algunos textos castellanos de los siglos XV y XVI. De fray Ambrosio Montesino son los versos siguientes, que se hallan en sus Coplas a la Natividad de la Virgen:

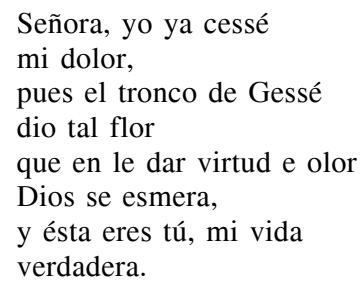

En el Cancionero espiritual editado en 1549 por Juan de Villaquirán, donde podemos encontrar divinizaciones que, en opinión de Wardropper (1954), siguen el estilo de finales del siglo XV, se hallan los siguientes versos referidos a la Virgen: 


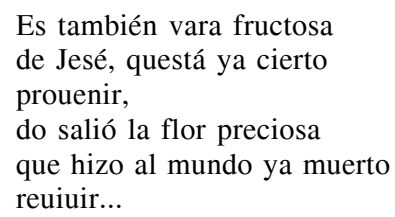

Por último, podemos señalar la obra del conquense Diego Cortés, que en la segunda mitad del siglo XVI nos daba los versos que siguen. Repárese en las analogías formales que guarda con nuestro poema:

De la raíz de Jesé

salió la graciosa vara

de más frescor y más clara

que en jamás será ni fue

\section{[II] Los profetas me demandan}

En cuanto al segundo poema, éste presenta una estructura de villancico. Los cuatro versos iniciales constituyen la cabeza, donde se enuncia el tema, y siguen tres estrofas compuestas por mudanza, verso de enlace y vuelta, seguida de los dos versos finales del estribillo. Su esquema sería el siguiente: $[-a-a] c d d c c a-a$.

Se hace uso del verso octosílabo, el más habitual en castellano y uno de los más frecuentes en las composiciones zejelescas. Al igual que en el texto [I], también aquí encontramos una gran regularidad métrica, con muy pocas licencias, pudiendo señalarse algunos casos de sinalefa. Alternan a lo largo del poema los versos oxítonos y paroxítonos. Del mismo modo, se combinan rimas agudas con rimas paroxítonas. En principio, la rima es en todos los casos consonante perfecta, incluso en la segunda estrofa («David» / «sí» / «decí»), pues en el verso 13 la $-d$ final seguramente no tendría valor fonológico si tenemos en cuenta los rasgos dialectales ya comentados anteriormente.

Los paralelismos métricos y estilísticos son los que contribuyen a dotar al poema de su estructura, especialmente algunas simetrías como las de los versos 21-23: «A mý sólo lo perguntan... Yo por respuesta les doy». A lo largo de las tres estrofas, se establece un diálogo entre Dios-Cristo y los profetas, que aguardan su llegada, siguiendo en todas ellas un mismo esquema: 1) Dios describe los lamentos y ruegos de los profetas. 2) Los profetas imploran a Dios. 3) Dios les pide que aguarden y anuncia su pronta llegada.

Es indudable que el autor de estos versos se apoya continuamente en el texto bíblico para describir a los profetas y elaborar sus intervenciones. En la primera estrofa, los lamentos de Jeremías guardan estrecha relación con varios versículos:

Quis dabit capiti meo aquam, et oculis meis fontem lacrimarum, et plorando die ac nocte interfectos filiae populi mei (Jeremías, 9, 1).

Deducant oculi mei lacrimam per noctem et diem (Jeremías, 14, 17). 
En la segunda estrofa, David ruega a Dios que incline los cielos, remitiendo a uno de los salmos:

Domine, inclina caelos tuos, et descende (Salmos, 143, 5).

Pero lo más interesante es que nuestro texto puede ponerse en relación con el ciclo medieval del Ordo Prophetarum, que remite a una ceremonia dramática desarrollada en una de las lecciones del oficio de maitines de la noche de Navidad o de la fiesta de la Circuncisión. En ella, después de la lectura de un sermón atribuido a San Agustín -Contra iudaeos, paganos et arrianos sermo de Símbolo-, se convocaba a diversos profetas y otros personajes de la tradición clásica y bíblica para que dieran testimonio de la venida de Cristo, siendo los más habituales Moisés, Isaías, Jeremías, David, Daniel...

Sin embargo, aunque el Ordo Prophetarum cuenta con no pocas versiones en la tradición europea, y muy especialmente en Francia, en el ámbito hispánico apenas conservamos testimonios. Sí tuvo cierta repercusión el conocido canto de la Sibila, que probablemente se desgajó de aquél, tal como señalaron Sepet y Young. Pero si exceptuamos el caso discutible de los Loores de Berceo, al que ya hicimos referencia, hemos de situarnos en las primeras décadas del siglo XVI para encontrar algunos testimonios de dicha representación en los que intervengan los profetas como protagonistas.

Uno de los ejemplos más antiguos conservados sería el de En una aldea para cantar la noche de Navidad, obra del mirobrigense Cristóbal de Castillejo, donde se incluye una adaptación al castellano del Judicii signum, tras la que intervienen diversos profetas manifestando su vaticinio sobre la venida de Cristo (Rodríguez Bordona, 1958: IV, 175-236). Beccaría (1988) sitúa su composición entre 1520 y 1550 , considerándolo el primer texto conocido del Ordo Prophetarum en España con la intervención de los profetas en lengua romance.

Más o menos por las mismas fechas debió de escribirse la Farsa del juego de cañas de Diego Sánchez de Badajoz (Pérez Priego, 1996), en la cual también se van sucediendo los profetas, introducidos por la Sibila, para dar testimonio y esperanza de la venida de Cristo. A dichas intervenciones sigue un estribillo que conmina, al igual que el de nuestro poema, a mantener la esperanza:

\footnotetext{
Quein espera non despera

Si esperança he vera.

Quein espera non despera

Si esperança he vera.
}

En esta obra, por cierto, podemos hallar insertos algunos pasajes que se extrajeron directamente de la liturgia: «Dirigite viam Domini / rectas facite in solitudine / Semitas Dei nostri». 
Por otro lado, si nos detenemos a analizar la copla inicial de nuestro poema, observaremos sorprendentes coincidencias con otras composiciones de mediados del siglo XVI, y no precisamente de carácter religioso. Ello corrobora que las influencias literarias traspasaron el ámbito litúrgico y religioso, recogiendo materiales de diversa procedencia. Así, por ejemplo, hallamos una clara analogía con los versos siguientes, recogidos por la profesora Margit Frenk (2003), que se encontraban en una carta de Perrenot a Felipe II fechada en 1562:

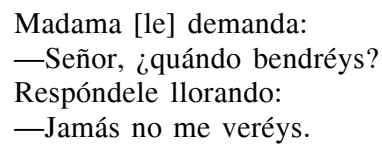

Pocos años después, en 1616, se publicaba el anónimo Baile curioso y grave, donde quedaban recogidos estos curiosos versos en forma de diálogo:

-Y mi bien, ¿cuándo vendréis?

—Lindo amor, no me aguardéis.

José María Alín (1968: 601-602) puso estos versos en relación con una canción recogida en el Cancionero sevillano de Nueva York, que fue estudiado y datado por la profesora Margit Frenk (1962) hacia 1568, fecha por tanto muy cercana a la copia de los textos que venimos analizando.

En ambos casos, las coincidencias con los versos iniciales de nuestro poema nos llevan a pensar en una divinización o «vuelta a lo divino» basada en una canción tradicional. No olvidemos que aproximadamente hasta 1580 se vivió el mayor auge de estas divinizaciones (Frenk: 1978), que fueron muy frecuentes en autores como Gómez Manrique, Íñigo de Mendoza, Ambrosio Montesino o Juan Álvarez Gato, y posteriormente en Juan del Encina o Lucas Fernández.

\section{BIBLIOGRAFÍA CITADA}

Alín, José María (1968). El cancionero español de tipo tradicional. Madrid: Taurus.

Beccaría Lago, $M^{a}$ Dolores (1988). «Sobre "En una aldea para cantar la noche de Navidad", de Cristóbal de Castillejo, y el drama litúrgico medieval», Dicenda. Cuadernos de Filología Hispánica (Arcadia. Estudios dedicados a F. López Estrada). II, 7, pp. 33-56.

Cátedra, Pedro Manuel (2000). «Liturgia, poesía y la renovación del teatro medieval», en Actas del XIII Congreso de la Asociación Internacional de Hispanistas. Madrid: Castalia, I, pp. 3-28.

Cátedra, Pedro Manuel (2005). Liturgia, poesía y teatro en la Edad Media. Estudios sobre prácticas culturales y literarias. Madrid: Gredos.

Corominas, Joan y José Antonio Pascual (1980). Diccionario crítico etimológico castellano e hispánico. Madrid: Gredos. 
Correas, Gonzalo (1967). Vocabulario de refranes y frases proverbiales. L. Combet (ed.). Burdeos: Institut d'Études Ibériques et Ibéro-Américaines de l'Université de Bordeaux.

Donovan, Richard B. (1958). The Liturgical Drama in Medieval Spain. Toronto: Pontifical Institute of Medieval Studies.

Frenk, Margit (1962). «El Cancionero sevillano de la Hispanic Society (c. 1568)», Nueva Revista de Filología Hispánica. 16, pp. 355-394.

Frenk, Margit (1978). Estudios sobre lírica antigua. Madrid: Castalia.

Frenk, Margit (2003). Nuevo corpus de la antigua lírica popular hispánica. Méjico: Facultad de Filosofía y Letras, Universidad Nacional Autónoma de México, El Colegio de México, Fondo de Cultura Económica.

Lapesa, Rafael (1981). Historia de la lengua española. Madrid: Gredos.

López-Morales, Humberto (1967). «Elementos leoneses en la lengua del teatro pastoril de los siglos XV y XVI», en Actas del Segundo Congreso Internacional de Hispanistas. Nimega: Instituto Español de la Universidad de Nimega, pp. 411-419.

Marchand, J. W. (1984). «Berceo the Learned: The Ordo Prophetarum in the Loores de Nuestra Señora», Kentucky Romance Quarterly. 31, pp. 291-304.

Pérez Priego, Miguel Ángel (1996). «La tradición representacional de la Sibila y la Farsa del juego de cañas, de Diego Sánchez», Criticón. 66-67, pp. 5-15.

Rodríguez Bordona, Jesús (1958). Obras de Castillejo. Madrid: Espasa-Calpe.

Sirera, Joseph Lluis (1992). «Diálogos de cancionero y teatralidad», en R. Beltrán, J. L. Canet y J. L. Sirera (eds.), Historias y ficciones: Coloquio sobre la literatura del siglo XV. Valencia: Universidad de Valencia, pp. 351-363.

Surtz, Ronald E. (1983). Teatro medieval castellano. Madrid: Taurus.

Turner, Victor (1986). Del rito al teatro. Bolonia: Il Mulino.

Wardropper, Bruce W. (1954). Cancionero espiritual de Juan de Villaquirán. Anónimo. 1549. Valencia: Castalia.

Fecha de recepción: 11 de junio de 2012

Fecha de aceptación: 7 de febrero de 2013 